PROCEEDINGS OF THE

AMERICAN MATHEMATICAL SOCIETY

Volume 138, Number 10, October 2010, Pages 3449-3454

S 0002-9939(10)10357-8

Article electronically published on April 13, 2010

\title{
ANALYTICALLY IRREDUCIBLE POLYNOMIALS WITH COEFFICIENTS IN A REAL-VALUED FIELD
}

\author{
A. GRANJA, M. C. MARTÍNEZ, AND C. RODRÍGUEZ
}

(Communicated by Bernd Ulrich)

\begin{abstract}
In this paper, we show a criterion of analytic irreducibility for polynomials with coefficients in a real-valued field. This generalizes previous criteria of Abhyankar as well as those of Granja.
\end{abstract}

\section{INTRODUCTION}

Throughout this paper, $K$ will be a field and $\nu$ will be a non-trivial valuation of real rank one on $K$. We will denote by $\widehat{K}$ the completion of $K$ with respect to the topology defined by $\nu$, by $\widehat{\nu}$ the unique extension of $\nu$ to $\widehat{K}$ and by $T$ an indeterminate over $\widehat{K}$.

Let $L$ be an algebraic extension of $K$. A natural question is: when does a valuation of finite real rank on $K$ have a unique extension to $L$ ?

Since an arbitrary algebraic field extension is a direct limit of finite extensions, we may assume that $L$ is finite over $K$. Moreover, it is sufficient to solve the problem when $\nu$ has real rank one. The general case can be reduced to the real rank case by means of composition of valuations. (See [ZS, Corollary 1, p. 55].)

Furthermore, since for purely inseparable extensions, $\nu$ has a unique extension, we can also assume that $L$ is a separable extension of $K$ and write $L=K(\alpha)$ for some algebraic and separable element $\alpha$ over $K$. In this case, let $P \in K[T]$ be the minimal monic irreducible polynomial of $\alpha$. Then $\nu$ has a unique extension to $K(\alpha)$ if and only if $P$ is irreducible in $\widehat{K}[T]$ (i.e. $P$ is analytically irreducible). (See $[\mathrm{E}$, Theorem (2.12), p. 16].)

In [HOS] an algorithm is given to determine the extensions of a real valuation to a simple algebraic extension. In particular, Corollary 66 of $[\mathrm{HOS}$ gives a characterization of when $\nu$ has a unique extension to $K(\alpha)$. Thus, the algorithm of [HOS] tests when a polynomial of $K[T]$ is irreducible in $\widehat{K}[T]$.

Here, we shall show a criterion of analytic irreducibility for polynomials of $K[T]$. (See Theorem 4.3 below.) In particular, if $\nu$ is discrete and $K$ is complete, our criterion is nothing but Theorem 4.7 of [G]. Moreover, if $K=F((X))$ is the field of the Laurent power series with coefficients in an algebraically closed field $F$ and $\nu$ is the usual order valuation on $X$, then our criterion also generalizes Abhyankar's criterion in $\mathrm{A}$.

Received by the editors September 28, 2009 and, in revised form, December 30, 2009.

2010 Mathematics Subject Classification. Primary 13B25, 12E05; Secondary 13A05, 13F30.

This work was partially supported by MCI, MTM2009-11433 and JCYL, LE003A09. 
The paper is organized in four sections including this introduction. Section 2 is devoted to reviewing some concepts about valuations on rings. In section 3 , we associate to each monic analytically irreducible polynomial $f \in K[T]$ a valuation $\nu_{f}$ on $K[T]$ such that $\nu_{f}$ is the unique extension of $\nu$ to $K[T]$ with $\nu_{f}(f)=\infty$. We show that $\nu_{f}(g)=\frac{\nu(\mathcal{R}(f, g))}{\operatorname{deg}(f)}$ for each $g \in K[T]$, where $\mathcal{R}(f, g)$ denotes the usual resultant of the polynomials $f$ and $g$. (See Proposition 3.1) The objective of the last section is to show the above-mentioned criterion of analytic irreducibility (Theorem 4.31).

\section{VALUATIONS ON RINGS}

In this section we review some concepts about valuations on rings.

Let $R$ be a commutative ring with unit and $\Gamma$ be a totally ordered abelian group. We extend $\Gamma$ to an ordered monoid $\Gamma \cup\{\infty\}$ by the rules $\infty+x=x+\infty=\infty$ for all $x \in \Gamma \cup\{\infty\}$ and $x<\infty$ for all $x \in \Gamma$.

A valuation on $R$ with values in $\Gamma$ is a map $\nu: R \longrightarrow \Gamma \cup\{\infty\}$ such that

(V1) $\nu(x y)=\nu(x)+\nu(y)$ for all $x, y \in R$,

(V2) $\nu(x+y) \geq \min \{\nu(x), \nu(y)\}$ for all $x, y \in R$,

(V3) $\nu(1)=0$ and $\nu(0)=\infty$.

If $\nu(R)=\{0, \infty\}, \nu$ is said to be trivial; otherwise $\nu$ is called non-trivial or proper. Moreover, the prime ideal $\nu^{-1}(\infty)$ of $R$ is called the support of $\nu$ and when $\Gamma=\mathbb{R}$ is the additive group of real numbers, $\nu$ is called a real valuation on $R$. In any case, the group of values $\Gamma_{\nu}$ of $\nu$ is the subgroup of $\Gamma$ generated by $\nu(R)-\{\infty\}$.

On the other hand, $\nu$ is called a Krull valuation on $R$ when $\nu^{-1}(\infty)=\{0\}$. In this case, $R$ is necessarily a domain and $\nu$ extends to a Krull valuation (also denoted by $\nu$ ) on the quotient field $K(R)$ of $R$ such that $\Gamma_{\nu}=\nu(K(R)-\{0\})$ is the group of values of $\nu$. Furthermore, $V_{\nu}=\{x \in K(R) ; \nu(x) \geq 0\}$ is the valuation ring associated to $\nu$, which is a local ring with maximal ideal $M\left(V_{\nu}\right)=$ $\{x \in K(R) ; \nu(x)>0\}$.

We also point out that any valuation $\nu$ on a ring $R$ induces a Krull valuation $\bar{\nu}$ on $R / \nu^{-1}(\infty)$. We say that $V_{\nu}=V_{\bar{\nu}}$ is the valuation ring associated with $\nu$ and we have $\Gamma_{\nu}=\Gamma_{\bar{\nu}}$.

We recall that for a Krull valuation $\nu$ on a local domain $R$, the real rank, or simply the rank of $\nu$ (denoted $\operatorname{rank}(\nu)$ ), is the Krull dimension of the ring $V_{\nu}$ and that if this dimension is finite, then $\operatorname{rank}(\nu)$ is the least integer $l$ so that $\Gamma_{\nu}=\nu(K(R)-\{0\})$ can be embedded as an ordered group into $\left(\mathbb{R}^{l},+\right)$ endowed with the lexicographic order. Hence, $\operatorname{rank}(\nu)$ depends only on the group of values of $\nu$. For details about the rank and other numerical invariants of Krull valuations, we refer to [ZS, Vol. II, Chapter VI, p. 50].

\section{VALUATION ASSOCIATED TO ANALYTICALly IRREDUCIBLE POLYNOMIALS}

Let $K$ be a field and $\nu$ be a non-trivial real valuation on $K$. Let us denote by $\widehat{K}$ the completion of $K$ with respect to the topology defined by $\nu$ and by $\widehat{\nu}$ the unique extension of $\nu$ to $\widehat{K}$. Let $T$ be an indeterminate over $\widehat{K}$.

We say that a polynomial $f \in K[T]$ is analytically irreducible if $f$ is irreducible in $\widehat{K}[T]$. Note that any analytically irreducible polynomial of $K[T]$ is an irreducible polynomial, but the converse is, in general, false. 
Now, we shall introduce some notation for monic analytically irreducible polynomials.

Let $f \in K[T]$ be an analytically irreducible monic polynomial and let $\alpha$ be a root of $f$ in some algebraic closure of $\widehat{K}$. Since $\widehat{K}$ is complete, there exists a unique extension, which we will denote by $\widehat{\nu}_{f}$, of $\widehat{\nu}$ to $\widehat{K}(\alpha)$. (See [, A, p. 127].) We shall also denote by $\widehat{\nu}_{f}$ the valuation on $\widehat{K}[T]$ given by $\widehat{\nu}_{f}(g)=\infty$ if $g \in f \widehat{K}[T]$ and by $\widehat{\nu}_{f}(g)=\overline{\widehat{\nu}}_{f}(\bar{g})$ if $g \notin f \widehat{K}[T]$, where $\bar{g}$ is the class of $g$ in $\frac{\widehat{K}[T]}{f \widehat{K}[T]}$. We point out that $\widehat{\nu}_{f}(g)=\bar{\nu}_{f}(g(\alpha))$.

Finally, we will denote by $\overline{\nu_{f}}$ (resp. by $\left.\nu_{f}\right)$ the restriction of $\overline{\widehat{\nu}_{f}}$ (resp. of $\left.\widehat{\nu}_{f}\right)$ to $K(\alpha)$ (resp. to $K[T]$ ). Then $\overline{\nu_{f}}\left(\right.$ resp. $\left.\nu_{f}\right)$ is the unique extension of $\nu$ to $K(\alpha)$ (resp. to $K[T]$ such that $\nu_{f}(f)=\infty$ ).

We can reduce the computation of $\widehat{\nu}_{f}$ (resp. $\nu_{f}$ ) to $\widehat{\nu}$ (resp. $\nu$ ) by means of the usual resultant of two polynomials $\mathcal{R}(P, Q)$ (see $[\mathrm{L}$, p. 200]). Namely, we have the following:

Proposition 3.1. With the above assumptions and notation, let $f \in K[T]$ be a monic analytically irreducible polynomial. Then $\widehat{\nu}_{f}(g)=\frac{\widehat{\nu}(\mathcal{R}(f, g))}{\operatorname{deg}(f)}$ for all $g \in$ $\widehat{K}[T]$, where $\mathcal{R}$ denotes the usual resultant ([L, p. 200]). In particular, $\nu_{f}(g)=$ $\frac{\nu(\mathcal{R}(f, g))}{\operatorname{deg}(f)}$ for all $g \in K[T]$.

Proof. Let $\alpha_{1}, \ldots, \alpha_{r}$ be the roots of $f$ in an algebraic closure of $\widehat{K}(r \leq \operatorname{deg}(f))$. Since $\widehat{K}$ is complete, there exists a unique extension $\overline{\widehat{\nu}_{f}^{\prime}}$ of $\widehat{\nu}$ to $\widehat{K}\left(\alpha_{1}, \ldots, \alpha_{r}\right)$. (See [R, A, p. 127].)

For $1 \leq i \leq r$, let $\sigma_{i}$ be a $\widehat{K}$-automorphism of $\widehat{K}\left(\alpha_{1}, \ldots, \alpha_{r}\right)$ such that $\sigma_{i}\left(\alpha_{1}\right)=$ $\alpha_{i}$. Then $\overline{\widehat{\nu}_{f}^{\prime}} \circ \sigma_{i}$ is a valuation of $\widehat{K}\left(\alpha_{1}, \ldots, \alpha_{r}\right)$ extending $\widehat{\nu}$ (see [R, I, $(\alpha)$, p. 118]). Therefore, ${\widehat{\widehat{\nu}_{f}^{\prime}}}_{f}=\overline{\widehat{\nu}_{f}^{\prime}} \circ \sigma_{i}$ and $\overline{\widehat{\nu}_{f}^{\prime}}\left(g\left(\alpha_{1}\right)\right)={\overline{\widehat{\nu}_{f}^{\prime}}}_{f}\left(\sigma_{i}\left(g\left(\alpha_{1}\right)\right)\right)=\overline{\widehat{\nu}_{f}^{\prime}}\left(g\left(\alpha_{i}\right)\right), 1 \leq i \leq r$.

We point out that if $\overline{\widehat{\nu}_{f}^{(i)}}$ denotes the restriction of $\overline{\widehat{\nu}_{f}^{\prime}}$ to $\widehat{K}\left(\alpha_{i}\right)$, then $\overline{\widehat{\nu}_{f}^{(i)}}$ is the unique extension of $\widehat{\nu}$ to $\widehat{K}\left(\alpha_{i}\right), 1 \leq i \leq r$. Hence, $\widehat{\widehat{\nu}}_{f}^{(1)}\left(g\left(\alpha_{1}\right)\right)=\overline{\widehat{\nu}_{f}^{(i)}}\left(g\left(\alpha_{i}\right)\right)$, $1 \leq i \leq r$.

On the other hand, we can write $f=\prod_{i=1}^{r}\left(T-\alpha_{i}\right)^{s_{i}}$, where $s_{i}$ is the multiplicity of the root $\alpha_{i}, 1 \leq i \leq r$. (Note that, in fact, $s_{i}=1$, if $f$ is a separable polynomial and $s_{i}=p^{s}$, otherwise, $1 \leq i \leq r$, where $p$ is the characteristic of $\widehat{K}$.) We have $\mathcal{R}(f, g)=\prod_{i=1}^{r}\left(g\left(\alpha_{i}\right)\right)^{s_{i}}$ (see [L], Proposition 8.3, p. 202). Thus,

$$
\begin{gathered}
\widehat{\nu}_{f}(\mathcal{R}(f, g))=\sum_{i=1}^{r} s_{i}{\overline{\widehat{\nu}_{f}^{\prime}}}^{\prime}\left(g\left(\alpha_{i}\right)\right)=\operatorname{deg}(f){\overline{\widehat{\nu}_{f}^{\prime}}}^{\prime}\left(g\left(\alpha_{1}\right)\right) \\
=\operatorname{deg}(f) \overline{\widehat{\nu}_{f}^{(1)}}\left(g\left(\alpha_{1}\right)\right)=\operatorname{deg}(f) \widehat{\nu}_{f}(g) .
\end{gathered}
$$

\section{Analytic CRITERION OF IRREDUCIBILITy}

With the assumptions and notation as in the above section, we shall reduce the analytic irreducibility of polynomials of $K[T]$ to that of monic polynomials of $V_{\nu}[T]$, where $V_{\nu}$ is the valuation ring associated to $\nu$. 
Let $f \in K[T]$ be an analytically irreducible polynomial with $\operatorname{deg}(f) \geq 2$ and $\alpha \neq 0$ be a root of $f$. Without loss of generality we can assume that $f$ is a monic polynomial and write $f=T^{d}+a_{d-1} T^{d-1}+\cdots+a_{0} \in K[T]$.

If $f \in V_{\nu}[T]$, then $\nu_{f}(T)=\overline{\widehat{\nu}}_{f}(\alpha) \geq 0$. Otherwise, $\overline{\widehat{\nu}}_{f}(\alpha)<0$ and $\bar{\nu}_{f}\left(\alpha^{-1}\right)>0$. Since $\alpha+a_{d-1}+a_{d-2} \alpha^{-1}+\cdots+a_{0}\left(\alpha^{-1}\right)^{d-1}=0$, we have $\overline{\widehat{\nu}}_{f}(\alpha) \geq 0$, which is a contradiction.

If $\nu_{f}(T)=\overline{\widehat{\nu}}_{f}(\alpha)<0$, then $\nu\left(a_{0}\right)<\nu\left(a_{i}\right), 1 \leq i \leq d-1$ and $\nu\left(a_{0}\right)<0$. Otherwise, there exists $0<s<d$ such that $\nu\left(a_{s}\right) \leq \nu\left(a_{0}\right)$ and $\nu\left(a_{s}\right)<0$. (Note that $f \in K[T]-V_{\nu}[T]$.) Since $\widehat{K}$ is a Henselian field and $f$ is an irreducible polynomial of $\widehat{K}[T]$, then $\nu\left(a_{s}\right) \geq((d-s) / d) \nu\left(a_{0}\right)$. (See $[\mathbb{R}$, Theorem 1, p. 128].) Therefore, $\nu\left(a_{0}\right)<0$ and since $\nu\left(a_{s}\right) \leq \nu\left(a_{0}\right)$, we get $\nu\left(a_{0}\right) \geq((d-s) / d) \nu\left(a_{0}\right)$, which is a contradiction.

Finally, we note that if $\nu_{f}(T)=\overline{\widehat{\nu}}_{f}(\alpha)<0$, then $\widetilde{f}=a_{0}^{-1} T^{d} f(1 / T) \in V_{\nu}[T]$ is a monic analytically irreducible polynomial. In fact, $\widetilde{f}$ is the minimal irreducible polynomial of $\alpha^{-1}$ over $K$ (and $\widehat{K}$ ). In this form, we must only give a criterion of analytic irreducibility for monic polynomials of $V_{\nu}[T]$.

Now, we shall introduce some more notation. Let $f \in V_{\nu}[T]$ be a monic analytically irreducible polynomial. We denote by

$$
\Omega_{l}\left(\nu_{f}\right)=\left\{\nu_{f}(g) ; g \in V_{\nu}[T] \text { monic with } \operatorname{deg}(g)=l\right\}
$$

and by $\omega_{l}\left(\nu_{f}\right)=\sup \left(\Omega_{l}\left(\nu_{f}\right)\right), 0 \leq l<\operatorname{deg}(f)$. Note that $\omega_{l}\left(\nu_{f}\right) \in \mathbb{R} \cup\{\infty\}$ and $0=\omega_{0}\left(\nu_{f}\right) \leq \omega_{1}\left(\nu_{f}\right) \leq \omega_{2}\left(\nu_{f}\right) \leq \cdots \leq \omega_{\operatorname{deg}(f)-1}\left(\nu_{f}\right) \leq \infty$.

Lemma 4.1. With the above assumptions and notation, let $f \in V_{\nu}[T]$ be a monic analytically irreducible polynomial. Then $\nu_{f}(\phi) \leq \nu\left(b_{h}\right)+\omega_{h}\left(\nu_{f}\right) \leq \nu\left(b_{h}\right)+\omega_{s}\left(\nu_{f}\right)$, for each non-zero polynomial $\phi=\sum_{i=0}^{s} b_{i} T^{i} \in V_{\nu}[T]$ with $1 \leq \operatorname{deg}(\phi)=s<\operatorname{deg}(f)$, where $h$ is the non-negative integer $0 \leq h \leq s$ such that $\nu\left(b_{h}\right)<\nu\left(b_{i}\right), h<i \leq s$ and $\nu\left(b_{h}\right) \leq \nu\left(b_{j}\right), 0 \leq j \leq h$; i.e., $h$ is the greatest index for which $\nu\left(b_{h}\right)=$ $\min \left\{\nu\left(b_{0}\right), \ldots, \nu\left(b_{s}\right)\right\}$.

Proof. Since $b_{h} \neq 0$, it is sufficient to show that $\nu_{f}\left(\left(b_{h}\right)^{-1} \phi\right) \leq \omega_{h}\left(\nu_{f}\right)$. Therefore, we can assume $b_{h}=1$ and $\nu\left(b_{h}\right)=0$.

Now, we proceed by induction on $s$.

Assume $s=1$. Then we have the following possibilities:

a) $h=s=1$. In this case $\phi=T+b_{0}$ with $\nu\left(b_{0}\right) \geq 0$ and $\nu_{f}(\phi) \leq \omega_{1}\left(\nu_{f}\right)$ by definition of $\omega_{1}\left(\nu_{f}\right)$.

b) $h=0<1$. In this case, $\phi=b_{1} T+1$ with $\nu\left(b_{1}\right)>0$ and $\nu_{f}(\phi)=0 \leq \omega_{1}\left(\nu_{f}\right)$. (Note that $\nu_{f}(\phi)=\widehat{\nu}_{f}(\phi(\alpha))=0$, where $\alpha$ is a root of $f$.)

Assume that the result is true for each $\phi \in V_{\nu}[T]$ with $\operatorname{deg}(\phi) \leq s-1, s \geq 2$.

If $h=s$, then $\nu_{f}(\phi) \leq \omega_{h}\left(\nu_{f}\right)$ by definition of $\omega_{h}\left(\nu_{f}\right)$; and if $h=0$, then $\nu_{f}(\phi)=0=\omega_{0}\left(\nu_{f}\right)$. (Note that $\nu_{f}(\phi)=\widehat{\nu}_{f}(\phi(\alpha))=0$, where $\alpha$ is a root of $f$.) Therefore, we can assume $0<h<s$, so $\nu\left(b_{s}\right)>0$.

Since $\omega_{s}\left(\nu_{f}\right)=\sup \left(\Omega_{s}\left(\nu_{f}\right)\right)$ and $\nu\left(b_{s}\right)>0$, let us fix a monic polynomial $g \in$ $V_{\nu}[T]$ such that $\operatorname{deg}(g)=s$ and $\nu\left(b_{s}\right)+\nu_{f}(g)>\omega_{s}\left(\nu_{f}\right)$ (for example, if $\omega_{s}\left(\nu_{f}\right)$ is reached, we may take $g$ to be such that $\left.\nu_{f}(g)=\omega_{s}\left(\nu_{f}\right)\right)$.

Let us write $\phi=b_{s} g+c_{s-1} T^{s-1}+\cdots+c_{0}$. Since $\nu\left(b_{s}\right)>0$ and $f, g \in V_{\nu}[T]$, $0=\nu\left(c_{h}\right)<\nu\left(c_{i}\right), h<i \leq s-1$ and $0=\nu\left(c_{h}\right) \leq \nu\left(c_{j}\right), 0 \leq j<h$. Therefore, $\nu_{f}\left(c_{s-1} T^{s-1}+\cdots+c_{0}\right) \leq \omega_{h}\left(\nu_{f}\right) \leq \omega_{s}\left(\nu_{f}\right)$ by the induction hypothesis. Hence, 
since $\nu_{f}\left(b_{s} g\right)>\omega_{s}\left(\nu_{f}\right)$, we have $\nu_{f}(\phi)=\nu_{f}\left(c_{s-1} T^{s-1}+\cdots+c_{0}\right) \leq \omega_{h}\left(\nu_{f}\right)$, and the proof is complete.

Lemma 4.2. With the above assumptions and notation, let $f \in V_{\nu}[T]$ be a monic analytically irreducible polynomial. Then the set $\Omega_{i}\left(\nu_{f}\right)$ is bounded above for $1 \leq$ $i<\operatorname{deg}(f)$, i.e. $\omega_{i}\left(\nu_{f}\right)<\infty$ for $0 \leq i \leq \operatorname{deg}(f)-1$. In particular, the set $\left\{\nu(\mathcal{R}(f, g)) ; g \in V_{\nu}[T]\right.$ monic with $\left.\operatorname{deg}(g)=i\right\}$ is also bounded above for $1 \leq i<$ $\operatorname{deg}(f)$.

Proof. Assume that $\Omega_{i}\left(\nu_{f}\right)$ is not bounded above for some $i<\operatorname{deg}(f)$ and that $\Omega_{l}\left(\nu_{f}\right)$ is bounded above for $0 \leq l<i$. In particular, $\omega_{i-1}\left(\nu_{f}\right)<\infty$.

Let us consider a sequence $\left\{\phi_{n}\right\}_{n \geq 1} \subset V_{\nu}[T]$ such that $\nu_{f}\left(\phi_{n}\right) \geq n$ and $\phi_{n}=$ $T^{i}+a_{i-1}^{(n)} T^{i-1}+\cdots+a_{0}^{(n)}, n \geq 1$. By Lemma 4.1, we have

$$
n \leq \nu_{f}\left(\phi_{n+1}-\phi_{n}\right) \leq \min _{0 \leq j \leq i-1}\left\{\nu\left(a_{j}^{(n+1)}-a_{j}^{(n)}\right)\right\}+\omega_{i-1}\left(\nu_{f}\right) .
$$

Therefore, $n-\omega_{i-1}\left(\nu_{f}\right) \leq \nu\left(a_{l}^{(n+1)}-a_{l}^{(n)}\right)$ for $0 \leq l \leq i-1$ and $n \geq 1$. So, $\left\{a_{l}^{(n)}\right\}_{n \geq 1}$ is a Cauchy sequence on $K$ with respect to $\nu, 0 \leq l \leq i-1$. Let us write $a_{l}=\lim _{n \rightarrow \infty} a_{l}^{(n)} \in \widehat{K}, 0 \leq l \leq i-1$ and $\phi=T^{i}+a_{i-1} T^{i-1}+\cdots+a_{0} \in \widehat{K}[T]$.

We have $\widehat{\nu}\left(a_{l}-a_{l}^{(n)}\right) \geq n-\omega_{i-1}\left(\nu_{f}\right), 0 \leq l \leq s-1, n \geq 1$. Since $\nu_{f}(T)=$ $\widehat{\nu}_{f}(T) \geq 0$, then $\widehat{\nu}_{f}\left(\phi-\phi_{n}\right) \geq n-\omega_{i-1}\left(\nu_{f}\right)$ and $\widehat{\nu}_{f}(\phi) \geq \min \left\{\widehat{\nu}_{f}\left(\phi-\phi_{n}\right), \nu_{f}\left(\phi_{n}\right)\right\} \geq$ $n-\omega_{i-1}\left(\nu_{f}\right), n \geq 1$. Hence, $\widehat{\nu}_{f}(\phi)=\frac{\widehat{\nu}(\mathcal{R}(f, \phi))}{\operatorname{deg}(f)}=\infty$ and $\widehat{\nu}(\mathcal{R}(f, \phi))=\infty$. Thus, $f$ and $\phi$ must have a common irreducible factor in $\widehat{K}[T]$, and since $f$ is analytically irreducible, $\phi \in f \widehat{K}[T]$ and $\operatorname{deg}(f) \leq \operatorname{deg}(\phi)$, which is a contradiction.

Next, we give our criterion of analytic irreducibility.

Theorem 4.3 (Criterion of Analytic Irreducibility). With the above assumptions and notation, let $P(T) \in V_{\nu}[T]$ be a monic polynomial. Then the following statements are equivalent:

(a) $P$ is analytically irreducible.

(b) The set $\{\nu(\mathcal{R}(P, Q)) ; Q \in K[T]$ monic with $\operatorname{deg}(Q)=i\}$ is bounded above for $1 \leq i<\operatorname{deg}(P)$.

(c) The set $\{\nu(\mathcal{R}(P, Q)) ; Q \in K[T]$ monic with $\operatorname{deg}(Q)=[\operatorname{deg}(P) / 2]\}$ is bounded above.

Here $[\operatorname{deg}(P) / 2]$ denotes the greatest integer s such that $s \leq \operatorname{deg}(P) / 2$.

Proof. $(a) \Rightarrow(b)$ follows from Lemma 4.2 and obviously $(b) \Rightarrow(c)$.

To see $(c) \Rightarrow(a)$, let us assume that $P$ is not analytically irreducible. We can write $P=P_{1} P_{2}$, where $P_{1}, P_{2} \in \widehat{K}[T]$ are monic polynomials, $h_{1}=\operatorname{deg}\left(P_{1}\right) \leq$ $[\operatorname{deg}(P) / 2]$ and $P_{1}(T)=T^{h_{1}}+a_{h_{1}-1} T^{h_{1}-1}+\cdots+a_{0}$ with $a_{j} \in \widehat{K}, 0 \leq j \leq h_{1}-1$.

For each integer $n \geq 0$ and for each $0 \leq j \leq h_{1}-1$, let us consider $a_{j}^{(n)} \in K$ such that $\widehat{\nu}\left(a_{j}-a_{j}^{(n)}\right) \geq n$ and write $P_{1}^{(n)}(T)=T^{h_{1}}+a_{h_{1}-1}^{(n)} T^{h_{1}-1}+\cdots+a_{0}^{(n)}$.

We recall that if $U_{0}, \ldots, U_{h_{1}}$ are indeterminates over $\widehat{K}[T]$, then

$$
\mathcal{R}(P, \underline{U})=\mathcal{R}\left(P, U_{0} T^{h_{1}}+U_{1} T^{h_{1}-1}+\cdots+U_{h_{1}}\right)
$$

is a homogenous polynomial on $U_{0}, \ldots, U_{h_{1}}$ of degree $\operatorname{deg}(P)$. 
Therefore, $\mathcal{R}\left(P, P_{1}^{(n)}\right)=\mathcal{R}\left(P, P_{1}\right)+\Lambda_{n}$ such that $\Lambda_{n} \in\left(a_{h_{1}-1}^{(n)}-a_{h_{1}-1}, a_{h_{1}-2}^{(n)}-\right.$ $\left.a_{h_{1}-2}, \ldots, a_{0}^{(n)}-a_{0}\right) V_{\widehat{\nu}}$, where $V_{\widehat{\nu}}$ is the valuation ring associated with $\widehat{\nu}$. Hence, $\widehat{\nu}\left(\Lambda_{n}\right) \geq n, n \geq 1$.

Since $\mathcal{R}\left(P, P_{1}\right)=0$, then $\nu\left(\mathcal{R}\left(P, P_{1}^{(n)}\right)\right)=\widehat{\nu}\left(\mathcal{R}\left(P, P_{1}^{(n)}\right)\right)=\widehat{\nu}\left(\Lambda_{n}\right) \geq n, n \geq 1$.

In particular, $\nu\left(\mathcal{R}\left(P, T^{[\operatorname{deg}(P) / 2]-h_{1}} P_{1}^{(n)}\right)\right) \geq n$ for $n \geq 1$ and the set

$$
\{\nu(\mathcal{R}(P, Q)) ; Q \in K[T] \text { monic with } \operatorname{deg}(Q)=[\operatorname{deg}(P) / 2]\}
$$

is not bounded above, which is a contradiction.

Remark 4.4. We point out that the irreducibility criterion given in Theorem 4.7 of [G] is a particular case of our Theorem 4.3. In fact, in [G], the valuation $\nu$ is assumed to be discrete and the field $K$ to be complete with respect to the topology defined by $\nu$. Moreover, if $K=F((X))$ is the field of the Laurent power series with coefficients in an algebraically closed field $F$ and $\nu$ is the usual order valuation on $X$, then our criterion also generalizes Abhyankar's criterion in $[$ ].

\section{REFERENCES}

[A] S.S. Abhyankar, Irreducibility criterion for germs of analytic functions of two complex variables. Adv. Math., 74, no. 2 (1989), 190-257. MR.997097(90h:32018)

[E] O. Endler, Valuation Theory. Springer-Verlag, Berlin, 1972. MR0357379 (50:9847)

[G] A. Granja, Irreducible polynomials with coefficients in a complete discrete valuation field. Adv. Math., 109, no. 1 (1994), 75-87. MR1302757 (95j:13006)

[HOS] F. J. Herrera Govantes, M. A. Olalla Acosta and M. Spivakovsky, Valuations in algebraic field extensions. J. Algebra, 312 (2007), 1033-1074. MR2333199 (2008e:12007)

[L] S. Lang, Algebra (Revised Third Edition). Graduate Text in Mathematics, 211, SpringerVerlag, New York, 2002. MR 1878556 (2003e:00003)

[R] P. Ribenboim, The Theory of Classical Valuations. Springer-Verlag, New York, 1999. MR.1677964 (2000d:12007)

[ZS] O. Zariski and P. Samuel, Commutative Algebra. Vols. I and II. Reprint of the 1958-60 edition, Graduate Texts in Math., 28-29, Springer-Verlag, New York, 1979. MR0384768 (52:5641), MR0389876 (52:10706)

Departamento de Matemáticas, Universidad de León, 24071-León, Spain

E-mail address: angel.granja@unileon.es

Departamento de Matemática Aplicada, Universidad de Valladolid, 47014-Valladolid, SPAIN

E-mail address: carmen@mat.uva.es

Departamento de Matemáticas, Universidad de León, 24071-León, Spain

E-mail address: mcrods@unileon.es 